\title{
Language and Speech Meets the HPCC Grand Challenge
}

\author{
Y.T. Chien \\ National Science Foundation \\ Washington, DC 20550
}

At a recent NSF workshop on speech understanding, Charles Wayne gave a presentation of Darpa's program on Speech and Language research. Towards the end of his presentation, he enlightened all of us with a brief outline of a possible new technology development program: High Performance Multi-lingual Speech and Text Processing. This is a very exciting and timely initiative, if it comes to that, as many of us in the federal government, NSF included, are preparing to launch new efforts to implement the President's High Performance Computing and Communications (HPCC) program beginning in this fiscal year.

The HPCC program is a real challenge and opportunity for all of us. Many in the research community , however, perceive "High Performance" as merely providing supercomputer cycles and services or developing new computer architectures and software for scientific or engineering research. As such language and speech research, they feel, will have very limited role to play. I beleive this perception is incorrect and unhealthy. In fact, language and speech is one of the most critical elements of the HPCC program for at least two reasons.

First, one of HPCC's goals is to address "Grand Challenge" problems. These are the fundamental problems in science and engineering, with broad economic and scientific impact, whose solution could be advanced by applying high performance computing techniques and resources. In this context, "language and speech" has been identified as one of those grand challenge problems, ranking it among such problems of national concern as weather prediction, drug design, superconductivity, human genome, and transportation systems and others $[1,2]$. The implication here is that computational speech and language is a critical problem for us to solve and its solution could be advanced by further research in high performance computing.

Another and perhaps more important reason, in my view, is that speech and language can help refine and drive HPCC research - ranging from architectures, software, algorithms and future novel applications as we move towards a knowledge intensive society. Here again,
Charles Wayne's list of possible objectives for future speech and language research tells us why: Rapid, effortless human-machine interaction vis speech, text, and other modalities, unlimited vocabulary speech recognition, natural speech syntheses, real time translation of speech/language, etc. Few other topics in the computing and communications field offer an equally rich set of research opportunities, with measurable goals and potential benefits.

At NSF, we are also beginning the implementation of the HPCC program. One of the components is a new "Grand Challenge" Applications Groups program focusing on cross-disciplinary research to address problems such as computational speech and language. Other similar HPCC initiatives may further develop, but their strategies and contents are likely more driven by the visions of the research community. Since HPCC is a multi-agency program, it will also provide a rare opportunity for Darpa and NSF to coordinate their goals and strategies to support research in speech and language. This is a challenge not only for the two agencies but for the research communities as well.

References:

1. "The federal High Performance Computing program", a report submitted to the Congress by the Office of Science and Technology, September, 1989.

2. "Grand Challenges 1993: High Performance Computing and Communications," a report by the FCCSET Committee to supplement the President's fiscal year 1993 budget. 